\title{
AUTOMATED ANALYSIS OF THE PRETERM NEONATAL CORTEX AT TERM EQUIVALENT AGE AND CORRELATION WITH COGNITIVE OUTCOME AT 1 YEAR CORRECTED AGE
}

\author{
A. Melbourne ${ }^{1}$, G.S. Kendall ${ }^{2}$, M.J. Cardoso ${ }^{1}$, P. San ${ }^{1}$, C. Hagmann ${ }^{2}$, A. Bainbridge ${ }^{3}$, S. Ourselin ${ }^{1}$, N. \\ Marlow $^{2}$, N.J. Robertson ${ }^{2}$ \\ ${ }^{1}$ Centre For Medical Image Computing, ${ }^{2}$ Academic Neonatology, University College London, ${ }^{3}$ Medical \\ Physics \& Bio-Engineering, University College London Hospital NHS Foundation Trust, London, UK
}

Background: Preterm birth $(<32$ weeks) is associated with differences in brain anatomical structure and adverse neurodevelopmental outcome. White matter volume and IQ have been shown to be reduced in expreterm infants in adolescence. Subtle differences in cortical folding at term equivalent age may be useful in predicting long-term neurodevelopmental outcome.

Methods: We segment grey matter (GM) and white matter (WM) using a maximum a posteriori expectation maximisation routine with adaptive priors. The GM/WM surface is extracted using a level set, and at every point we define the local shape-index, describing how cup-like or saddle-like the surface is (fig1A), and the curvedness, describing the distortion of the surface relative to that of a flat sheet. The distributions of each index may be summarised in a histogram (fig1B) and we use the histogram entropy as a potential biomarker.

Results: 39 infants without obvious structural abnormalities on MRI had brain volumes and cortical folding analysed from T1-weighted images. WM volume correlates significantly with gestation at birth $(\mathrm{p}<0.05)$. Shape index and curvedness did not correlate with gestation at birth. Shape index correlates with combined cognitive / language outcome at 1 year using the Bayley III scales of infant development (fig1C).

Conclusion: Cortical folding analysis may be useful, possibly in combination with white matter volumes in the development of biomarkers of neurodevelopmental outcome.

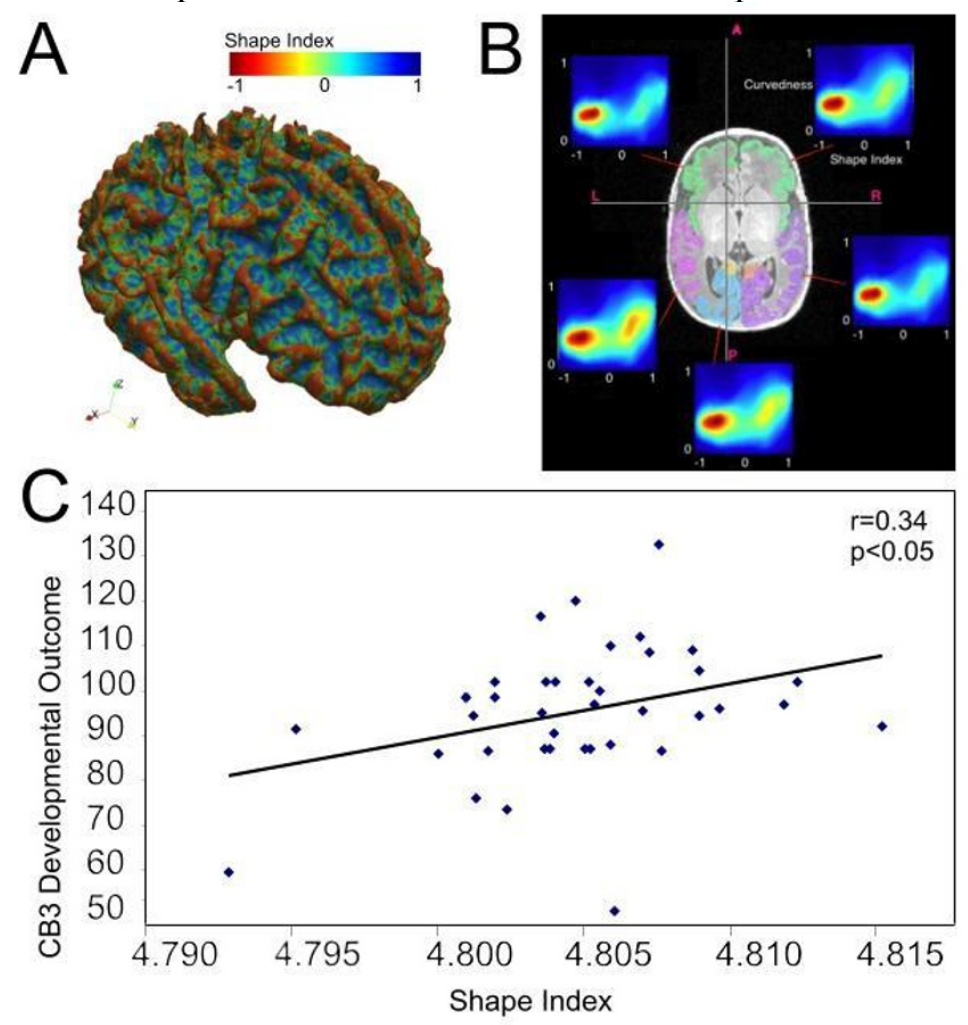

Figure 1 A: GM/WM interface coloured by shape-index B: Population average local surface histograms of curvedness and shape index overlaid on single subject GM parcellation C. Correlation between shape index \& combined cognitive/language outcome. [Figure1] 